2

\title{
Consideraciones acerca de la Ciencia como Cultura
}

\section{Considerations on Science as Culture}

\author{
DAVID NAVARRO \\ Universidad Industrial de Santander y \\ Universidad Autónoma de Bucaramanga [Colombia] \\ dnavarro0574@gmail.com \\ ORCID ID: http:/ / orcid.org/0000-0001-5016-73
}

\section{Resumen}

El presente trabajo consiste en una aproximación a la ciencia considerada como siendo en primera instancia un producto histórico y cultural. Dicha consideración no impide que la ciencia pueda ser también evaluada en su posición frente a otras formas culturales ofreciendo como resultado un serio cuestionamiento del relativismo cognitivo. En la línea de las tesis del pensador inglés Ernest Gellner realizamos una ponderación racionalista del conocimiento científico y su valor para la sociedad contemporánea.

Palabras clave: ciencia, cultura, Gellner, razón, relativismo.

\section{Abstract}

The present work consists of an approach to science considered as being in the first instance a historical and cultural product. This consideration does not prevent science from also being evaluated in its position against other cultural forms, resulting in a serious questioning of cognitive relativism. Along the lines of the theses of the English thinker Ernest Gellner, we carry out a rationalist weighing of scientific knowledge and its value for contemporary society.

Key words: science, culture, Gellner, reason, relativism. 


\section{INTRODUCCIÓN}

La modernidad se ha caracterizado por haber trazado una línea divisoria entre los terrenos de la teoría y de la práctica, así lo hizo desde su nacimiento a través de la gran figura de René Descartes. Nosotros queremos ensayar en este breve texto a llevarle la contraria a la modernidad -y de paso mucho más a la posmodernidad- para explorar si echando mano de la práctica, en el lugar y momento adecuados, es posible dar por sentada una forma de conocimiento como tajantemente superior a todas las demás en la historia de la humanidad y, por la misma vía, si es a su vez posible extraer desde el campo de la cognición algunas consecuencias para el terreno de la práctica, o lo que es decir, para el de la moral, diluyendo por tanto las fronteras modernas entre una y otra con el propósito de vislumbrar nuevas conexiones entre el camino de la ciencia y alguna posible forma de la sociedad en nuestro mundo actual, unas que podamos definir en una doble vía: negativamente, por lo que no habría de ser pero que se ha vuelto tendencia y que, adelantando un poco el meollo, consiste en una especie de condena por razones de vergüenza, una condena de la ciencia por su origen y por su poder, o también en devaluarla a ser un saberdiscurso sin más entre otros de su misma categoría. Pero también queremos trazar unos nexos positivamente, en este caso ligados a los ideales de libertad y justicia, máximos legados de la llustración.

\section{2. ¿UNA MORAL CIENTIIFICA O UNA BASE MORAL PARA LA CIENCIA?}

A propósito del gran filósofo francés que acabamos de mencionar, resultaría útil para empezar nuestra reflexión el volver la mirada a la interesante discusión que nos plantea su escurridiza idea de "la más alta y la más perfecta moral" mencionada en la Carta-Prefacio a sus Principios Filosóficos, pues precisamente su posibilidad, como él la concibe, estaría estrechamente vinculada al desarrollo de las ciencias. La esperanza cartesiana de que los seres humanos llegásemos a alcanzar tal desarrollo de las ciencias que nos permitiese deducir de ellas todas las verdades, incluso en el terreno de la moral, en sus textos parece muy laxa, si no es que literalmente utópica. Esto es precisamente porque el avance en nuestro ámbito moral se encontraría bajo esta visión extrañamente supeditado al científico y más bien coronando la verde proeza arbórea como el culmen del avance de la mecánica y de la medicina que son las otras dos ramas del árbol del conocimiento cartesiano. Esta imagen del árbol ha dado, por cierto, no poco que pensar y en un aspecto nos resulta un tema precioso para nuestro trabajo: ¿es la moral una rama de la misma jerarquía que la física o la medicina? Discutiremos esto unas líneas más abajo y planteamos aquí mismo una pregunta adicional: ¿es realmente tan extraño afirmar que nuestra moral esté involucrada con el desarrollo científico?

Podemos avanzar sobre de la naturaleza de esa divisoria que trazaba Descartes entre teoría y práctica pues, según lo expresan algunas de sus escuetas líneas sobre el tema, al parecer su ideal [¿utopía?]' es que no fuese más que provisional, es decir, del mismo talante que la moral de la que los hombres debían servirse hasta que se pudiese concebir una de carácter científico, o sea, de validez universal: tras siglos de mancomunados esfuerzos podría ser que hasta la moral tuviese un carácter científico. Sin embargo, bien leído, el filósofo francés nos enseña mucho más

$1 \quad$ Resulta bastante tentador pensar que Descartes admitiría no tener ninguna certeza acerca de la posibilidad de alcanzar una moral de tal perfección precisamente porque en su momento el mismo filósofo no podía tenerla según sus mismos criterios: esa certeza solo podría ser el producto de un desarrollo de las ciencias en su máximo grado, que en todo caso vendría al pasar de los siglos. También es posible que la moral le resultase inapropiada en últimas para un tratamiento matemático-deductivo a la manera de la física, como lo es en general lo correspondiente al compuesto dual cuerpo-alma. Es una discusión abierta a los estudiosos del filósofo francés. 
acerca de la conexión deseable entre la ciencia y la moral que de las razones por las cuales se puede o no tener una certeza razonable de alcanzar una tan elevada moral. Y es que la ciencia, especialmente a través de la tecnología, podría hacernos la vida mucho más feliz. O, al menos, más vivible. Aunque la ciencia al parecer no tiene que ver con el sentido de la vida, pues de ella no emana contenido alguno del que servirnos para nuestro hacer práctico, lo que sí parece un anhelo legítimo es que la ciencia sirva al noble propósito de facilitarnos una existencia tan digna como sea posible y, por qué no, hasta placentera. Para ello, nuestra propuesta es en cierto sentido una inversión del proyecto cartesiano: apliquemos al máximo nuestras mejores capacidades morales [sin más remedio que las que tengamos por provisión] al campo de la ciencia: la "más alta y perfecta ciencia" ha de construirse sobre un determinado cimiento moral. Y ese cimiento vendría ya ganado en buena parte para nosotros por la llustración, por ello no es necesario esperar siglos, lo podemos hacer ya. Es más, es imprescindible hacerlo de inmediato y con las herramientas disponibles: por una parte con los valores ilustrados de libertad e igualdad y por otra con la razón, digámoslo cartesianamente, sobresaliente entre ellas. Para especificar mejor cuál haya de ser ese sostén moral de la ciencia hemos en primer lugar de comprender a la propia ciencia como cultura y evaluar qué estatuto le corresponde.

\subsection{La ciencia como cultura}

Es preciso realizar una mínima aclaración terminológica previa para evitar posibles confusiones: cuando hablemos de "cultura" lo haremos de la forma más general, es decir, como aquella que incluye toda producción humana tanto material como intelectual. Más adelante y en el momento pertinente también haremos uso del término en plural "culturas" para referirnos a cosmovisiones, es decir, a vínculos y formas de interpretar el mundo, a saberes y a formas de conocer compartidos por un grupo poblacional específico. En esta misma línea trataremos la moral como lo que se refiere al terreno de los valores y del sentido en tanto que son comunitarios y delimitados por comunidades y territorios. Finalmente, siguiendo a Descartes, nos referimos a lo teórico como a lo relativo al conocimiento propio de las ciencias y a lo práctico como lo respectivo a las costumbres y las decisiones que se deben tomar en la vida cotidiana ${ }^{2}$. Por lo tanto, para nosotros la cultura conceptualizada en su sentido amplio involucra tanto la teoría como la práctica. Y nuestra cuestión al respecto de estas últimas, teoría y práctica, es: ¿es que pueden caminar realmente la una sin la otra, o no será más bien que una y otra se constituyen recíprocamente?

Uno de los grandes interrogantes que deben afrontar las ciencias humanas es el que nos cuestiona acerca del valor de las distintas culturas que a lo largo de la historia de la especie humana le han servido tanto de vínculo comunitario y soporte espiritual como de fuentes de sentido e interpretación de la realidad. El alcance de tal cuestionamiento podemos empezar a comprenderlo si tenemos en cuenta que nuestro acceso al mundo está irremediablemente mediado por los instrumentos que nos ofrece la cultura. Mismos que históricamente han ido cambiando y en alguna ocasión afinando: adquirimos ocasionalmente más capacidad de control sobre la naturaleza que nos rodea y a más de esta mayor es la verdad de la creencia en comparación con otras en su relación con la realidad. He ahí la sencilla clave de un difícil acertijo: la ciencia en tanto que saber es un producto humano e histórico, fruto de una compleja imbricación de

$\overline{2}$ Recordemos precisamente que el motivo de la escisión cartesiana fue su prudencia en el terreno de la práctica, donde recomendaba una moral bastante conservadora hasta poder disponer de la más alta moral [la que llegará de la mano de la ciencia]. Otra cosa fue su espíritu revolucionario en el terreno teórico, del cual despachó sin miramientos a la tradición como enemiga de la verdad. 
contingencias y avatares y no de ningún plan maestro, así que la ciencia es cultura. Sin embargo y de forma creciente la ciencia ha conseguido establecer una relación tal con la realidad que ya no es comparable con otras formas de conocimiento. En este sentido la ciencia tiene, por el control que muestra sobre la realidad que conoce, un privilegio epistémico sobre las demás culturas y saberes tradicionales o contemporáneos con que se la quiera comparar y también un privilegio sobre la verdad si admitimos que la verdad tiene que ver con la capacidad de describir, predecir y afectar la realidad sobre la que versan las creencias. Dado que la validación de todo conocimiento requiere de la formulación de unas reglas, y dado que esas reglas son expresadas en relación a un determinado contexto, su independencia total no es posible. Por ello la teoría se afirma en la práctica, la capacidad de control refrenda la teoría y al conectar con el mundo hace lo que la teoría por sí misma no alcanza: nos ofrece un criterio adicional de objetividad, al menos el máximo al que podemos aspirar, porque tampoco es este control empírico, claro está, absoluto, pero es lo que nos exige absolutamente eso sí una, digamos, "ética" del conocimiento. Incluso para aquellos que tienen en gran estima (muy por encima de su aprecio a la verdad) la relación saber-poder, parece que resulta inevitable conceder a la ciencia un lugar claramente aventajado. De lo que hablamos es en definitiva de una característica fundamental y definitoria de la modernidad, esta consiste en que la legitimidad de las creencias depende no ya de la consolidación o de la estabilización de un cuerpo de creencias como de la especificación de un procedimiento de validación que les conceda algún grado, el mayor posible, de externalidad y con ello de objetividad. Este es el juego de las teorías "selectoras"3, usando el término que emplea el filósofo y sociólogo inglés Ernest Gellner [1974] en su magnífica obra Legitimation of Belief. Estas "selectoras" son hijas de los siglos XVII y XVIII que habrían conseguido establecer criterios que, jatención!, trascienden las sociedades y sus culturas. Pero, quizás estamos yendo demasiado rápido. Vamos a detenernos un poco y replantear nuestro problema de la siguiente manera: ¿estamos elogiando una forma cultural particular que en realidad no es más que una entre otras de sus semejantes a las que se debería la misma ponderación? o, lo que es lo mismo: ¿es posible hablar en nuestros días de un relativismo en sentido cognitivo?

\subsection{Cultura científica y relativismo}

Adelantamos una paradójica respuesta: el relativismo es posible con respecto de todas las culturas menos una. El relativismo solamente se plantea de forma aceptable en verdades morales, o como hemos definido más arriba, si se quiere al hablar de "culturas" en un cierto sentido, en el de una interpretación del mundo mediada por tradiciones, pero no en las verdades de la esfera cognitiva. Es decir, si al hablar de relativismo cultural, el término cultura se refiere a costumbres, valores o una concepción particular de la vida buena, entonces aplica. Pero si lo usamos para designar formas de conocimiento, como por ejemplo el médico, no se vale. Y vamos a continuación a sustentar este argumento.

Los abordajes más habituales de la problemática del relativismo cultural discurren en dos sentidos. La vía de carácter propiamente relativista nos dirá que no hay forma de medir objetivamente -por ello tampoco comparativamente- el valor de una determinada cultura porque eso implicaría el que se pudiese evaluar dicha formación desde una perspectiva externa a toda cultura y eso no es

3 Entre las más valoradas por el autor cabe mencionar el empirismo [selector a partir de datos] y el materialismo [selector a partir de explicaciones]. Su función es establecer los criterios bajo los cuáles una creencia es legítima, pero de partida no respetan su pedigree o su estatus tradicional: ni su arraigo, ni su longevidad o su extendida aceptación, por ejemplo. De hecho, al enfrentarse al sistema de creencias tradicional, las teorías selectoras cambiaron el mundo. 
humanamente posible. Todo evaluador y los criterios mismos del juez son en definitiva productos culturales y por tanto no pueden pretender ningún privilegio epistémico sobre otras formas de conocer o de comprender el mundo. Podemos decir que todo sistema de creencias, ya sean religiosas, científicas, morales, políticas, etc., son productos culturales y, en tanto que tal, ninguno de ellos puede hacer un juicio objetivo sobre otro sistema dada la inconmensurabilidad de cada sistema particular. Líneas abajo nos extenderemos más para analizar esta forma de respuesta, pero podemos ya adelantar que es la propia de la visión posmoderna de la cultura y también de la etnografía.

Una segunda vía, asume que hay en efecto una forma cultural que ha conseguido situarse más allá de las culturas en general y cuya forma de conocimiento es la única forma legítima de conocer y, por lo tanto, podría ofrecernos unos criterios valorativos y la posibilidad de jerarquizar las creencias de acuerdo con ellos. Esta es una tesis muy fuerte que, dada la orientación posmoderna de la gran mayoría de académicos en la actualidad, parece estar condenada por ser prepotente, positivista y anti-relativista. Vamos a denominar esta posición como "fundamentalismo racionalista" de acuerdo con el autor que nos sirve de referente para nuestra explicación a partir de este punto, el ya mencionado filósofo inglés Ernest Gellner. Tendremos que detenernos a evaluar más profundamente cada una de las respuestas que hemos presentado hasta ahora solo con un bosquejo para poder llegar al fondo de la cuestión. Para este propósito seguimos los argumentos de Gellner [1994].

\subsubsection{Relativismo}

En primer lugar, diremos que una posición relativista es aquella que afirma el igual estatus de todo sistema de creencias. Estos son entendidos por la teoría posmoderna ${ }^{4}$ como sistemas simbólicos que construyen el sentido, orientan la acción de las personas, posibilitan una forma de conocer y comprender la realidad y, en algunos casos más radicales, hasta asumir la verdad y la realidad como efectos culturales.

El relativismo de las últimas décadas postula al significado como el principal objeto de estudio de las ciencias humanas y sociales pues de él dependen la configuración del orden social y la valoración de lo que haya de ser la verdad (o su minusvaloración se diría mejor). La realidad y todos sus elementos han de ser aprehendidos mediante los sistemas de significación. Se entiende que los significados constituyen la realidad o al menos que esta se manifiesta a través de ellos como única vía posible. Una consecuencia directa de este postulado es que toda verdad ha de ser hermenéutica, es decir, una interpretación que es fruto de una mirada mediada por un sistema de significados local y que no puede tener el acceso a una única verdad universal. Los significados hacen las cosas, por lo tanto, las cosas son un doble producto cultural: los significados son productos culturales en primer lugar y de ellos surgen las cosas como segunda producción cultural. El significado no es neutro y puede ser portador de un ejercicio de dominación al incluir tanto existencia como clasificación y estatus, la realidad es significada y jerarquizada al mismo tiempo, de tal manera que cuando nuestros propios pensamientos, sentimientos y sensaciones son impregnados, como inevitablemente lo son desde nuestra infancia, por la cultura, se subjetivizan esencialmente y no puede ser de otra manera. A su vez ellos subjetivizan para el sujeto su entorno, la realidad y la verdad, y no puede ser de otra manera. No existe un

$4 \quad$ Utilizamos una referencia muy general a la concepción de la posmodernidad para señalar algunas de sus características más comunes. Una definición exhaustiva del término requeriría un trabajo más extenso que desborda nuestros objetivos. 
investigador, sea cual fuere su campo de estudios, que pueda alcanzar la objetividad, pues el investigador es él mismo un producto cultural que no puede trascender su subjetividad.

El respeto cultural y la simetría entre las diversas interpretaciones del mundo son consecuencias del subjetivismo. Frecuentemente encontraremos en sus defensores una admiración por la diversidad, por lo excluido e incluso por lo marginal y lo extraño. Para ellos, el esfuerzo encaminado a presentar una realidad como objetiva o la simple generalización es el trabajo de la dominación y suele corresponderse con el de una cultura particular. Es imprescindible abandonar la pretensión de alcanzar una concepción de la verdad o de la realidad únicas, como lo quisieran las ciencias están en el origen de la modernidad y que en realidad obedecían al proyecto de dominación y supremacía de una cultura narcisista y prepotente. Debemos admitir que solo hay interpretaciones. La subjetividad es inextricable en dos sentidos: en la construcción del mundo por parte de los sujetos y en la elaboración de las teorías acerca de ese mundo que están en los textos. Está presente tanto en la comunidad (lugar del sujeto investigado) como en el científico [el sujeto generador del conocimiento]. La omnipresencia de la subjetividad tiene también como consecuencia el igualitarismo político, es decir, la necesidad de una igualdad en la diversidad pues no hay un criterio que pueda conceder privilegios a una determinada interpretación sobre otra en lo que las concepciones del bien o del orden social se refiere. La hermenéutica y la cultura se alían para dar lugar a una concepción social a la que le ha costado mucho expresarse en un programa de acción política, pero eso es harina de otro costal ${ }^{5}$ : la igualdad en la diversidad. Como efecto perverso hemos de señalar que el relativismo en tanto principio normativo también justifica, al menos lógicamente, cualquier forma de despotismo de carácter local, pues eso es también parte de la "diversidad" cultural.

Para recapitular, reflejemos de forma muy breve las principales consecuencias del argumento relativista:

$\checkmark$ Es necesario renunciar a la concepción de la verdad única: toda verdad es hermenéutica, no hay verdades que trasciendan las culturas.

$\checkmark$ Ninguna cultura es mejor o peor que otra, no disponemos de herramientas extra-culturales para establecer jerarquías. Las culturas son inconmensurables y un relativista lógicamente debería ser un nihilista (otra cosa es que no asuma esta consecuencia por los motivos que sea].

$\checkmark$ Ninguna cultura puede someterse a ningún criterio externo, pues sencillamente no hay criterios neutros y extra-culturales. Igualmente, las distintas formas de conocimiento, dado que son ellas mismas herramientas culturales, deben ser respetadas en términos de igualdad.

La posmodernidad es el movimiento filosófico que durante el siglo XX ha sido el apologeta de este posicionamiento. En términos de Gellner, la epistemología relativista de la posmodernidad

$5 \quad$ Chantal Mouffe y Ernesto Laclau podrían ser sugerentes pensadores en esta línea de investigación. Estos autores trabajan en una idea de construcción democrática radical de consenso como forma de movilización política frente a una general tendencia de la posmodernidad a la abstención frente a cualquier proyecto de universalización de ideales, frente a los cuales ellos -los posmodernos- movilizan todas sus sospechas de tal manera que solo se han permitido legitimar lo "común" o las prácticas de lo "comunitario" a niveles muy locales, ojalá tribales, y mucho mejor y más acreditado, por ejemplo en Latinoamérica, si pueden trazar su genealogía hasta algún momento prehispánico. Entonces ya es motivo de toda clase de elogios. 
da su apoyo incondicional a toda teoría del "respaldo" ${ }^{\text {, }}$, resultando en consecuencia reaccionaria por excelencia. La más lamentable consecuencia de esta faz reaccionaria del relativismo posmoderno, es decir, su afecto exacerbado por todo lo tradicional, lo local y lo subjetivo, en fin, su romanticismo delirante [en detrimento de lo universal, lo objetivo y la verdad: por demás, lo que caracteriza la ciencia] es que da al traste con las intenciones emancipatorias que podía enarbolar la posmodernidad. Un desastre en definitiva es lo que cabe esperar de esto de tirar por la borda los grandes logros de la ilustración [el impulso de la igualdad y la libertad como valores universales] y la modernidad [una racionalidad científica o una ética de la cognición que da un valor objetivo al conocimiento] en nombre de un subjetivismo paranoico al que le encanta coquetear con la irracionalidad.

\subsubsection{La falsedad del relativismo cognitivo}

Una vía alternativa a la del relativismo es precisamente la que podemos encontrar en el racionalismo radical de Ernest Gellner. Siguiendo sus argumentos expuestos tanto en Postmodernismo, Razón y Religión [Gellner, 1994] como en Razón y Cultura [Gellner, 1992] podremos apreciar cuáles serían las principales tesis a favor de una especial jerarquía al respecto de una cultura en particular: la científica.

Hay una afirmación muy seria en la obra de Ernest Gellner que hay que poner en el punto de partida y que debemos justificar acto seguido: la realidad y la verdad son únicas y hay una forma de conocerlas que trasciende las culturas: la ciencia. Y hemos de decir más aún, sujétese usted a la silla estimado lector, porque esa forma de conocer que trasciende culturas es a su vez un producto cultural. Nada mejor que enunciarlo claramente, todo conocimiento es hijo de una cultura y la ciencia, lo es, sin más. Pero una vez que, teniendo en cuenta esto y pudiendo, lo mejor que podamos, reconstruir la historia de los azares por los cuales finalmente la ciencia vio la luz, hemos de reconocer también lo que es innegable: sus conocimientos son válidos y comunicables universalmente y han transformado de una manera incomparable la sociedad humana. Es en efecto un tipo de conocimiento excepcionalmente poderoso que ha modificado el terreno de la producción y la economía en general, las comunicaciones, la industria militar, la salud, etc. Ha incluso dejado ver su capacidad de operar ya no sobre nuestro entorno sino sobre la naturaleza propiamente humana, suscitando todo tipo de cuestionamientos tanto en el uno como en la otra. $Y$ es que estos conocimientos no respetan el orden cultural ni la moralidad, siendo en muchas ocasiones contrarios a ellos. La particular concepción de la naturaleza que tiene la ciencia, es decir, la de una única naturaleza que se rige por un único y estable sistema de leyes, es aceptada hasta por los investigadores que se muestran más sensibles al relativismo: resultaría conmovedor que un etnólogo, por muy de talante geertziano que se pusiese, afirmara que allí donde ha hecho su investigación la magia sí funciona a diferencia del resto del mundo conocido. En realidad, lo que hace el etnógrafo es describir las prácticas rituales como efectuadas en la misma naturaleza del científico y ahí limita su amor a la diversidad por el no menos valioso aprecio de la sensatez. Sin embargo, nunca son suficientes las precauciones y los llamados de atención en este sentido, pues quien escribe estas líneas ha tenido que sufrir la experiencia de ver y oír como desde la propia academia de filosofía se incita a ensayar a curarse enfermedades tan serias como el cáncer con magia, recomendando el vodoo por ejemplo, cosa que en un país como

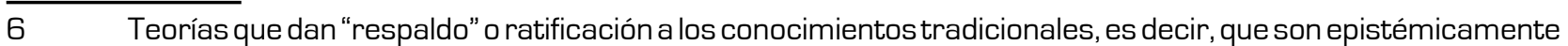
conservadoras y su función es la de ratificar un statu quo. Es notable entre ellas el papel de la filosofía lingüística como heredera de la propuesta del segundo Wittgenstein. Son la contrapartida de las teorías "selectoras" características de la modernidad. 
Colombia, donde he vivido muy recientemente la experiencia que menciono, no tiene ninguna gracia, pues ante la verdadera necesidad del pueblo de acceso a recursos médicos a todos los niveles aparecen los intelectuales de la posmodernidad predicando que si lo que hay a mano es magia, pues bienvenida sea ya que se merece ser igualitariamente ponderada, en su diversidad, con respecto del tratamiento científico. Esta bendición académica de la diversidad se convierte perversamente en una bendición al atraso, a la precariedad y a la muerte. Podríamos dibujar una recomendación que de alguna manera da la vuelta a la situación que originalmente enfrentó Descartes: a los entusiastas de la posmodernidad les decimos que bien cabe una revolución de la diversidad para nuestra vida práctica, ojalá acompañada de una actitud siempre crítica e irónica hacia las propias costumbres, pero recomendamos prudencia y sensatez en el terreno teórico, mismo en el que se han de seguir las buenas directrices de la tradición más poderosa que tenemos: la ciencia.

La ciencia natural tiene la capacidad de generar un conocimiento acumulativo y altamente consensuado. Su superioridad en la práctica es innegable y sus logros no son el tesoro de una determinada cultura sino de toda la humanidad. A la vez resulta que son aplicables en toda ella pues, por ejemplo, las vacunas no distinguen entre "occidentales" y "orientales", no prefieren curar a quienes profesan esta o aquella creencia religiosa, etc. En efecto, el método científico genera conocimientos que trascienden las culturas y es avalado por su ética del conocimiento: una avasalladora conexión entre control y verdad legitima sus creencias y anula cualquier vestigio de relativismo cognitivo. De esta manera, el relativismo cognitivo es falso pues la auto-vigilante censura epistemológica que selecciona rigurosamente las creencias de la ciencia tanto como la prueba de la experiencia a la manera de una estricta ética obran la diferencia. Es el ejercicio de una racionalidad que es selectora de creencias, que además se apoya en una experiencia que ha de ser posible repetir si se requiere y, por supuesto, debe ser públicamente examinable y observable: esta es la vía para trascender la mirada local, el ojo de la cultura "esta" o "aquella". Si existe un conocimiento "otro" que satisfaga estos estándares, pues sencillamente ya no es un otro, pasa a ser parte de la ciencia, venga de donde venga, haga lo que haga, fuese su objeto el que fuese ${ }^{7}$. La validez de una explicación se desliga del estatus del sujeto que la propone y pasa a depender de su posibilidad de ser corroborada. Esto es la modernidad. Podemos presentar el contenido de esta ética del conocimiento [o de una racionalidad científica] en unos pocos puntos muy bien sintetizados por Carmen Ors-Marqués a partir del artículo de Ralph Schroeder [1996], From the Big Divide to the Rubber Cage:

$\checkmark$ La validez de las creencias no se vincula a un cuerpo de ideas sustantivas sino al método a través del cual hemos llegado a esas creencias. Los sistemas de creencias que no se someten a las normas de validez o procedimientos del método son inaceptables como conocimiento.

$\checkmark$ El conocimiento científico es acumulativo, esto depende del consenso que genera entre los investigadores y, por otro lado, de que por norma siempre debe estar sometido a revisión y descarte a la luz de las sucesivas revisiones.

$\checkmark$ Las revisiones se realizan a partir de la información empírica, los datos, de forma que

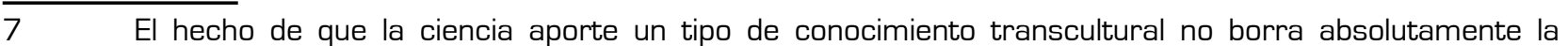
subjetividad ni tampoco la convierte en infalible. Esto no debe confundirse con ninguna forma de cientificismo. Ahora, en nuestros tiempos esta transculturalidad que hace parte del conocimiento científico debería invitarnos a dejar de unir como si fuesen lo mismo las dos palabras "ciencia" y "occidental" que se ponen juntas con tanta frecuencia y tendenciosamente para dar a entender que intrínsecamente la ciencia hace parte del ejercicio de la dominación de Occidente. Esto es falso, la ciencia es culturalmente neutra así se use a menudo y lamentablemente de otra manera. 
cualquier conjunto de creencias o hipótesis debe ser susceptible de ser puesta en tela de juicio partir de estos datos.

$\checkmark$ Los datos deben ser lo más "atómicos" posibles, la evidencia empírica debe tratarse en la forma de piezas aislables o separables y no como paquetes previamente atomizados.

$\checkmark$ Todos los datos deben ser tratados por igual, a ninguno puede dársele un status especial.

$\checkmark$ También los observadores, al menos en principio o en las mismas condiciones, son iguales.

$\checkmark$ Los datos han de servir para postular estructuras y someterlas a revisión.

$\checkmark$ Las estructuras se entienden como modelos simétricos que explican cómo sus partes están vinculadas y que se aplican a toda la evidencia por igual sin excepciones.

$\checkmark$ Las estructuras explican un conjunto de fenómenos que puede ser subsumido en ellas y son reproducibles.

$\checkmark$ Estas estructuras han de ser transparentes en el sentido de que las vinculaciones entre los elementos que explican pueden revisarse y comprobarse.

$\checkmark$ Por último, como los datos, las estructuras son accesibles al escrutinio de todos los investigadores [Ors Marqués, 2011].

Sin embargo, de acuerdo con Gellner, de todo esto no se puede extraer ningún criterio que nos sirva de orientación para la búsqueda de un orden social sostenible ni para dotar de sentido a la vida humana. A lo largo de la historia sí que este estilo de pensamiento ha coadyuvado a cuestionar ciertos regímenes, por ejemplo los absolutistas ${ }^{8}$, pues la ciencia requiere un alto grado de libertad para la investigación y bien sabemos que sus resultados pueden desafiar las cosmovisiones tradicionales de este o aquel credo, pero de sus hallazgos no se deduce cuál habría de ser nuestra forma de coexistir. Ahora, esto sí hay que tenerlo en cuenta, en la actualidad existe una gran asimetría entre las sociedades que han conseguido acoger con éxito los frutos de esta forma de saber y las que no. Poseerla es un anhelo legítimo de toda sociedad y es difícil comprender cómo y por qué los relativistas, que son tan sensibles a toda diferencia cultural, se han mostrado ciegos ante la más abismal de todas.

\section{UNA BREVE REFLEXIÓN FINAL ACERCA DE LA CIENCIA COMO SENTIDO Y EMANCIPACIÓN}

Ante esta ceguera, quisiéramos concluir con algunas reflexiones acerca de los vínculos entre la ciencia, la cultura y la moral. Gellner insiste en que no es tarea de la ciencia dotar de sentido alguno a la vida humana, pero nosotros planteamos que tal vez eso no sea tan tajantemente cierto pues ¿no es acaso la búsqueda de la verdad y del conocimiento la tarea más propia de sí que el ser humano ha emprendido desde que ha existido?, ¿no es por tanto la ciencia, esto es, la ciencia tomada no como producto sino como actividad, algo profundamente lleno de un sentido humano? Nos parece que las respuestas son necesariamente afirmativas y que, además, negar que la ciencia sea portadora de sentido sea tanto como negar la mayor de que la ciencia sea cultura. Ahora, si bien el conocimiento que nos ofrece la ciencia es transcultural y se sobrepone a cualquier perspectiva relativista, su oferta de sentido no lo es más que a un nivel personal, pero

8 Entonces, podemos apreciar que la ciencia al menos en un sentido general sí que sugiere algún modelo de sociedad: le es muy duro convivir con los absolutismos y se desarrolla mejor cuanta más libertad exista para la crítica. 
sí puede ser válida para un ser humano cualquiera, con tal que elija la ciencia o ella a él ${ }^{9}$, tanto como para otros es donadora de sentido esta o aquella fuente cultural, llámese religión, tradición, costumbre, etc. Y luego, los científicos también hacen comunidades.

La situación contemporánea es cartesiana en el sentido de que asumimos una moral permanentemente provisional y por lo tanto revisable en todo momento. Nuestro deber es ser críticos con nuestra propia cultura, asumirla, pues, con una actitud irónica y crítica, alejados de todo dogmatismo. Podemos asumir que la provisionalidad es la característica del ser humano. Pero, por otra parte, debemos una racional sensatez a procurar y alentar todo desarrollo científico que pueda mejorar nuestras condiciones de vida y en especial las de los habitantes más desfavorecidos de nuestro planeta, las actitudes posmodernas que tachan sin más a la "ciencia occidental" como un saber que es él mismo el ejercicio de la dominación imperialista o eurocentrista no son más que una nueva forma de pensamiento reaccionario que se viene extendiendo con peligrosa fuerza desde mediados del siglo XX. Si bien la ciencia, por contingencias históricas ha tenido su cuna en Europa, su saber y beneficios no son en nuestro tiempo el efecto cultural exclusivo de las creencias de una determinada población: lo que debemos solucionar es la injusticia en su distribución, no atacar recalcitrantemente sus capacidades.

Volvamos una última vez al problema de si la justificación de la superioridad que se arroga la cultura científica es razonable. La cuestión es que podría dar la impresión de que se está presentando como universalmente válida una particularidad cultural, con sus propias categorías, significados y criterios de verdad. Anteriormente hemos señalado la imposibilidad de juzgar una cultura bajo los criterios de otra, pues en tanto que culturas están en posición de igualdad y asumir lo contrario no es otra cosa que el ejercicio de la dominación. Pero ¿no puede ser que la posición relativista se aleje de la realidad para, habiéndose desconectado totalmente de ella, afirmar la igualdad de todo sistema simbólico [cultural]? Nos parece claro que no disponemos de una justificación a priori de la superioridad cognitiva de la ciencia, pero la racionalidad del argumento gellneriano, que a una históricamente decantada y refinada metodología selectiva de las creencias suma sus poderosos efectos prácticos, verificables, contrastables y repetibles, resulta difícilmente objetable. A esto hemos de añadir que nos parece muy recomendable que en aquellos lugares del mundo donde la asimetría causa los mayores sufrimientos, el realismo sea la norma y la guía de la acción social en miras de la justicia y la libertad, si es que estos valores se pueden considerar como universalmente deseables [seguramente lo serán para aquellos cuya ausencia marca sus vidas] son sin duda los que deben acompañar el ejercicio de la ciencia. El creer que la emancipación es ante todo una batalla que se ha de librar en el terreno de los significados es tan solo un lujo, o hasta una pose, para los académicos cuya situación está más bien asegurada y para sociedades donde la cultura científica, y he ahí una inmensa ironía, ha logrado alcanzar un alto desarrollo y la población se beneficia de ello. No se trata de negar que hay dominación conceptual, pero creemos que es importante y urgente atender, y luchar decididamente contra, los efectos de las diferencias económicas y la coerción política, siendo los suyos los efectos más devastadores. Sin creencias compartidas y bien fundadas ¿cómo luchar? Y es que no nos queda claro cómo podría un relativista ofrecernos una crítica del absolutismo, el machismo o cualquier otra forma de dominación presente en otra cultura. Por otra parte, ya hemos anotado que de la cultura científica no cabe esperar una fórmula moral o política, pero también nos parece que de acuerdo con la realidad sí es pertinente señalar que desviar la atención y tratar de encausar

9 Pues tanto vale para la ciencia como para otras transmisiones culturales una cierta imposición o involuntariedad, por ejemplo, desde un ámbito familiar o social, etc. 
las esperanzas de emancipación de los más desfavorecidos exclusivamente hacia el terreno de la diversidad y de lo local, descuidando la necesidad de construir proyectos de liberación universalizables, es un desacierto. El progreso técnico y económico, la igualdad y la justicia en estos ámbitos, son demandas insoslayables para enarbolar en las luchas sociales pues están en la raíz de muchas otras desigualdades y la ciencia debe ser apreciada como una herramienta de un inmenso potencial emancipador que debe ser firmemente encauzado por los irrenunciables ideales de la ilustración: la ciencia puede combatir el hambre, la ciencia puede combatir la enfermedad, la ciencia puede combatir la pobreza, la ciencia es lo contrario de la ignorancia. Lo que la ciencia pueda hacer por mejorar nuestro mundo depende de nosotros. Hacer que pueda se nos presenta como un deber.

\section{REFERENCIAS}

Gellner, E. [1994]. Razón y cultura. Madrid: Editorial Síntesis.

Gellner, E. [1992]. Posmodernismo, razón y religión. Barcelona: Paidós.

Gellner, E. (1974). Legitimation of Belief. Londres: Cambridge University Press.

Ors-Marqués, C. [2011). Razón y cultura en la filosofía de Ernest Gellner. [Tesis doctoral]. Universitat de València.

Schroeder, R. [1996]. From the Big Divide to the Rubber Cage: Gellner's Conception of Science and Technology. Poznan Studies in the Philosophy of the Sciences and the Humanities, 48, 427-444. 